\title{
Monitoreo de la pesquería del bacalao de profundidad (Dissostichus eleginoides): observadores científicos y sus desafíos
}

\author{
Monitoring of the patagonian toothfish fishery \\ (Dissostichus eleginoides): challenges of scientific observers
}

Renato Céspedes ${ }^{1}$, Cristian Vargas ${ }^{1} \&$ Luis Adasme $^{1}$

\section{Resumen}

El Instituto de Fomento Pesquero desempeñó un papel fundamental en el desarrollo de la pesquería de bacalao de profundidad (Dissostichus eleginoides Smitt, 1898) al sur del paralelo $47^{\circ}$ L.S., ejecutando desde inicios de los años noventa la investigación que permitió consolidar esta pesquería e impactar positivamente a la actividad económica de la XII Región de Magallanes, al sumar un recurso pesquero con alto valor agregado. Esta pesquería al sur del $47^{\circ}$ $\mathrm{S}$ ha contado con programa de monitoreo de levantamiento de datos biológicos y pesqueros para fines de administración y evaluación de la pesquería desde su origen, sustentado en registros tomados por observadores científicos embarcados en la flota pesquera de palangre. Los datos disponibles han sido clasificados en bitácoras de pesca, muestreos de talla de las capturas y muestreos biológicos pesqueros para conocer la composición de edad de las capturas y la condición reproductiva. En este sentido, el presente trabajo muestra los principales desafíos del programa de monitoreo de la pesquería, que al tener características de ser permanente $y$ oportuna la toma de datos biológicos pesqueros, también tiene entre sus metas lograr representar la dinámica espacio-temporal de la actividad de pesca y los patrones biológicos del recurso, siendo este un insumo clave para determinar el estado de situación de la pesquería, en donde la interacción biológica con los mamíferos (orcas y cachalotes) que predan sobre la captura de bacalao de profundidad, agrega al programa de monitoreo mayores desafíos a su investigación orientado a mejorar el conocimiento de la pesquería como sistema socio-ecológico.

\section{Palabras clave:}

pesquerías, programa de monitoreo, bacalao de profundidad, observador científico, palangre, orca, cachalote

\begin{abstract}
The IFOP (Instituto Fomento Pesquero) performed an important role in the development of the fishery of the Patagonian Toothfish (Dissostichus eleginoides Smitt, 1898) at the south of parallel $47^{\circ} \mathrm{S}$, executing the research from the early $90^{\prime}$ s that allowed this fishery to established and positively impact the economic activity in the 12 th Region of Magellan, adding the fishing resources with a high added value. This fishery (at the south of $47^{\circ} \mathrm{S}$ ) has been supported with a monitoring program of recording of biological and fishing data for administration and evaluation purposes of the fishery since its beginning, supported by data taken by scientific observers shipped in the fishing fleet of longline, classified in fishing's log, sample of size of the captures and fishing biological sampling to know the reproductive condition and the age composition of catches. In this sense, this work shows the main challenges of the monitoring program of the fishery, by having characteristics
\end{abstract}

\footnotetext{
Departamento de Evaluación de Pesquerías, Instituto de Fomento Pesquero (IFOP). Almte. Manuel Blanco Encalada 839, Valparaíso, Chile. $\longrightarrow$ renato.cespedes@ifop.cl
} 
of being permanent end timely the taking of the fishing's biological data, it also has as goal to achieve in representing the time and space dynamic of the fishing activity and patterns of biological resources, being this as a bais the key to determine the state fishery situation, where the biological interaction with the mammals (killer whales and sperm whales) that depredate to $t$ the capture of Patagonian Toothfish, it adds to the monitoring program mayor challenges to its research oriented to improve the fishery knowledge as a socio-ecological system.

\section{Key words:}

fisheries, monitoring program, patagonian toothfish, scientific oberserver, longline killer whale, sperm whale

\section{INTRODUCCIÓN}

Como antecedentes generales de la distribución y patrones biológicos de bacalao de profundidad es posible mencionar que el recurso es explotado en toda su distribución circumpolar Antártica, que comprende aguas antárticas del hemisferio sur, incluyendo los océanos Pacífico Suroriental, Atlántico e Índico, en áreas circundantes a las plataformas peninsulares de islas subantárticas como las Islas Malvinas, Georgia del Sur, Príncipe Eduardo, Bouvet, Kerguelen, Heard, Macquerie (Galleguillos et al. 2008). Mediante técnicas genéticas y química de otolitos se determinó la existencia de una unidad poblacional del bacalao de profundidad en la plataforma sudamericana (Galleguillos op cit.), que comprende Perú, Chile, Argentina e Islas Malvinas.

Antes de los años noventa los desembarques de la pesquería de bacalao de profundidad (Dissostichus eleginoides Smitt, 1898) fueron explicados principalmente por la pesca artesanal de la zona centro sur de Chile. Sin embargo, a inicios de los noventa producto de generar una actividad de pesca alternativa a la pesca industrial orientada a merluza del sur (Merluccius australis) en la zona austral, el IFOP inició los primeros estudios exploratorios dirigidos a bacalao de profundidad con barcos palangreros al sur del paralelo $47^{\circ} \mathrm{S}$. Los buenos resultados de rendimientos de pesca fueron la base para el desarrollo de una importante pesquería en la XII Región de Magallanes de Chile.

El bacalao de profundidad es un pez abisobentónico. En aguas chilenas se han capturado ejemplares hasta los $2.500 \mathrm{~m}$ de profundidad (Young et al. 1998), en donde los caladeros más importantes se ubican entre los 1.000 y $1.800 \mathrm{~m}$, presentando una estratificación por tamaño en relación con la profundidad, en donde los peces de menor tamaño están en aguas menos profundas (Young et al. 1999).

Esta especie es un desovante sincrónico (un evento de desove anual, Young op cit.). Young et al. (1992), señalan que el desove de bacalao de profundidad se produciría entre junio y agosto entre los $53^{\circ}$ y $57^{\circ} \mathrm{LS}$, etapa en la que existe una veda biológica para la especie (Decreto Supremo 273/96). Los hábitos alimenticios lo caracterizan como un depredador de alto nivel trófico. Estudios recientes en el área al sur del $47^{\circ}$ L.S. mostraron que la importancia de la dieta fueron las presas del orden gadiforme, como Antimora rostrata y Macruronus magellanicus, seguido de pejerratas, cefalópodos y crustáceos (Gálvez et al. 2014).

Desde un punto administrativo, la pesquería de bacalao de profundidad se subdividió en el paralelo del $47^{\circ} \mathrm{S}$. Al norte de dicho paralelo para la pesca artesanal y al sur de ella principalmente para la pesca industrial. Sobre esta última área se ha efectuado un permanente monitoreo de los indicadores biológicos y pesqueros, orientados al conocimiento de la dinámica de la pesquería, del recurso y la base de información para la evaluación de stock y el manejo de la pesquería.

En este sentido, el monitoreo de la pesquería ha tenido desafíos que han ido de la mano con el desarrollo de la pesquería de bacalao de profundidad al sur del $47^{\circ}$ L.S. La necesidad de información científica para la toma de decisiones y los presupuestos para investigación, en donde los Observadores Científicos que están embarcados son pieza clave para construir y sustentar el conocimiento de la pesquería.

El objetivo de este trabajo es presentar el estado actual del monitoreo de la pesquería al sur del paralelo $47^{\circ} \mathrm{S}$ basado en la toma de datos 
efectuado por los Observadores Científicos del IFOP, conjuntamente con los desafíos que se han abordado recientemente en colaboración con los usuarios de la pesquería y los desafíos de la toma de datos ante la demanda de requerimientos de investigación.

\section{Enfoque metodológico}

Este artículo tiene el contexto de una revisión del monitoreo de la pesquería que fue presentado en el "1er Seminario de Bacalao en Magallanes 2015" desarrollado entre el 24 y 25 de septiembre de 2015 en la ciudad de Punta Arenas, que dentro de su finalidad estuvo orientado a generar un debate del estado conocimiento de esta pesquería e identificar sus brechas. Bajo este enfoque, la presente revisión en forma resumida presenta el estado actual del monitoreo de los datos biológicos pesqueros y los desafíos que posiblemente se pueda ver enfrentados ante un escenario de demanda de conocimiento.

Luego el trabajo tiene el enfoque metodológico que en base a la información o los resultados que genera el programa de monitoreo de la pesquería de bacalao de profundidad, estos mostraran las situaciones que se ha visto enfrentada este programa y los impactos que ha generado en modificar el registro de los datos pesqueros, en donde cambios como los aparejos de pesca y la interacción de mamíferos en la pesquería han marcado adecuaciones importantes en el desarrollo de ella.

La fuente de información de los resultados provienen de la propia Fuente IFOP (monitoreo), como de estadística de desembarque de Sernapesca.

Monitoreo de la

pesquería al sur del $47^{\circ}$ L.S:

Toma de datos con Observadores Científicos

El área de estudio de la pesquería de bacalao de profundidad comprende las aguas exteriores al sur del paralelo $47^{\circ} \mathrm{S}$ e incluyendo áreas aledañas con aguas argentinas (Fig. 1).

$\mathrm{Al}$ inicio de esta pesquería, en los años noventa, se registraron los máximos desembarques en el área al sur del $47^{\circ}$ L.S., empleando el palangre español o tradicional como aparejo de pesca (Fig. 2). En este período el monitoreo se orientó a recopilar las bases de datos biológicos pesqueros de la pesquería que permitieron las primeras medidas de administración, como la veda biológica por patrones reproductivos, como también las primeras evaluaciones de stock que usaban series temporales de captura por unidad de esfuerzo (CPUE) como índice de abundancia, como también las estructuras de edad de las capturas.

En este período, los observadores científicos registraron bitácoras de pesca, muestreos de longitud de las capturas y los muestreos biológicos (recolección de escamas/ otolitos para estudios de edad y gónadas para condición reproductiva). Este sistema de colección de datos que en general se mantiene hasta la actualidad. En el caso de la edad se colectaron las escamas para estudios de la edad, pero a partir del año 2007 los estudios de edad se basan en la colección de los otolitos.

Durante los años noventa, la flota estuvo compuesta por una flota palangrera fábrica y otra flota palangrera hielera (Fig. 3), aspecto que generó una mayor complejidad en la cobertura, en donde el Observador Científico tuvo en la flota palangrera fábrica la mayor disposición de los armadores en los embarques y habitabilidad. Sin embargo, a partir del año 2005 solo ha operado la flota palangrera fábrica.

Luego, el estudio de monitoreo ha estado enfrentado desafíos principalmente a requerimientos de la administración. Uno de ellos ha sido contar con mayor cobertura espacio-temporal en la flota que opera sobre el recurso con el propósito de mejorar la toma de información biológica pesquera dada la señal descendiente del indicador de abundancia en el área al sur del $47^{\circ}$ L.S. (Wiff et al. 2006); período que también se generaron otros estudios sobre la pesquería y patrones biológicos en bacalao de profundidad financiados por el Fondo de Investigación Pesquera.

Durante este período reciente, los usuarios de la pesquería se vieron enfrentados al incremento de la interacción de mamíferos (orcas y cachalotes) en la pesca, que predan sobre la captura del recurso, afectando 


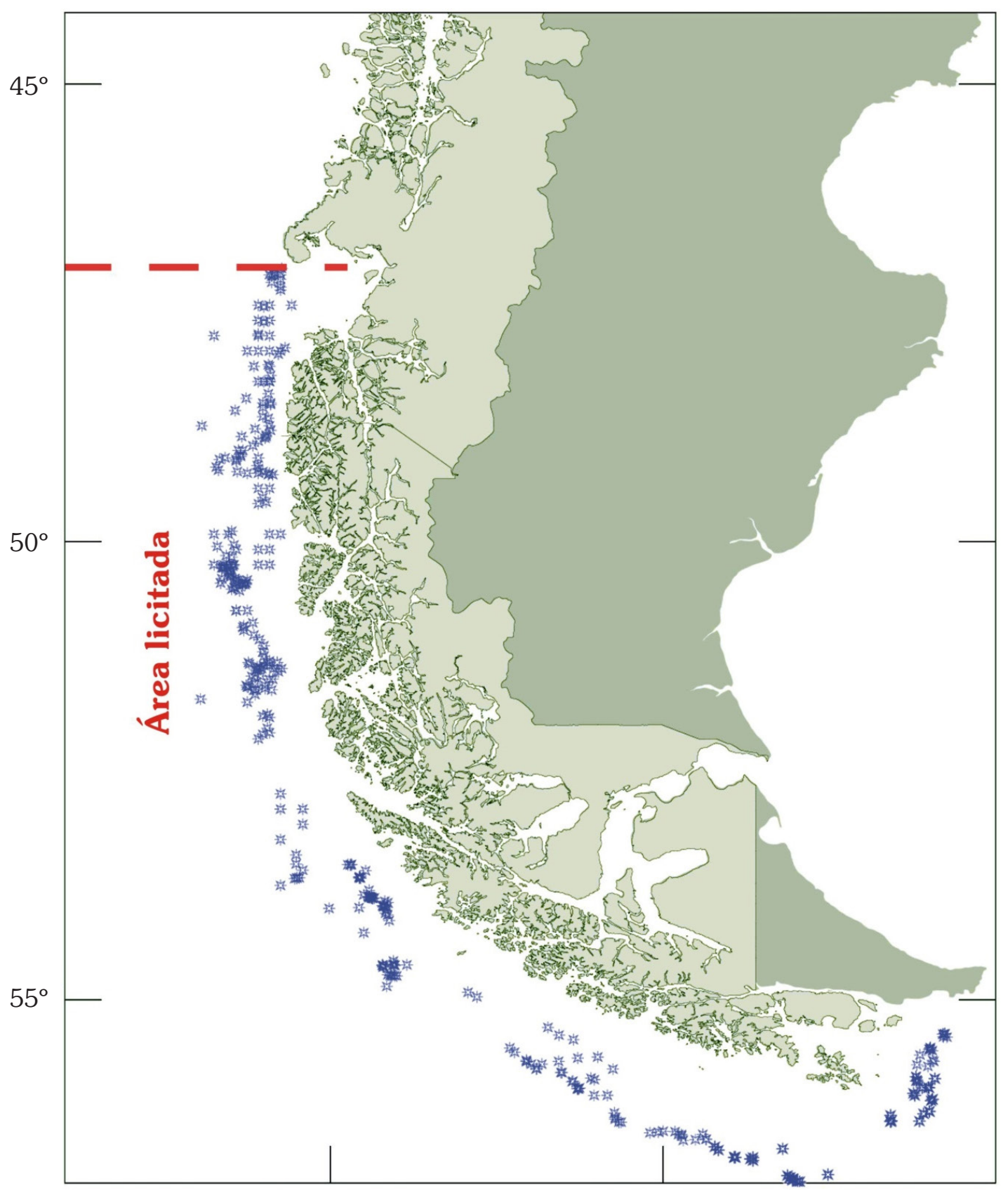

Fig. 1. Mapa geográfico de operación de la pesquería industrial austral en bacalao de profundidad. Los puntos indican la localización geográfica de los lances de pesca dirigidos al recurso en el año 2014. Fuente IFOP.

directamente con los rendimientos de pesca. Por otro lado, producto de convenios internacionales la administración inició los requerimientos de conocimiento respecto de la interacción de las aves marinas en pesquerías que empleaban el palangre como aparejo de pesca.

En el año 2006 los usuarios de la pesca de bacalao de profundidad iniciaron una modificación del aparejo de pesca al denominado palangre "cachalotero" (ver Fig. 2), para reducir el efecto 


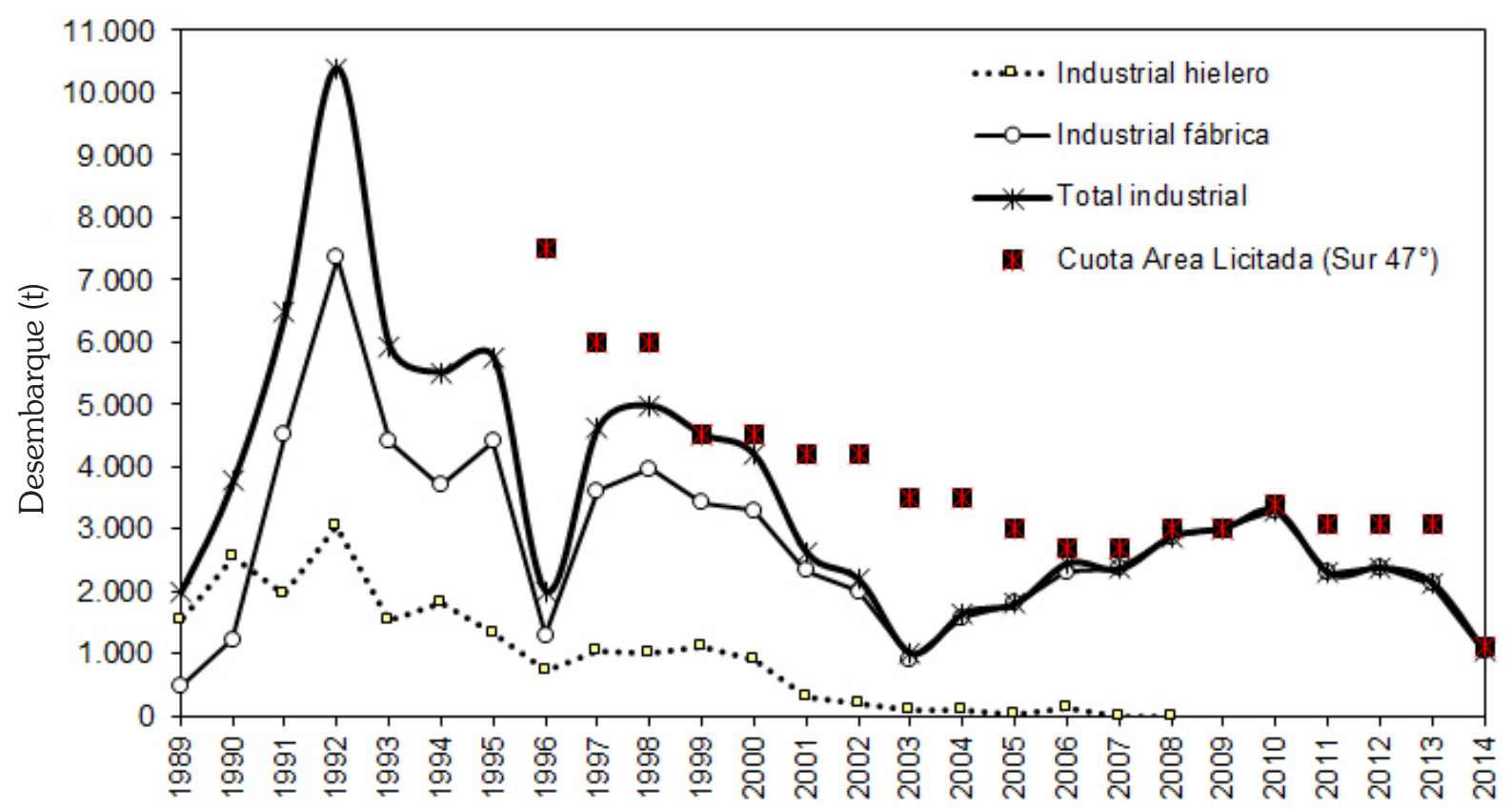

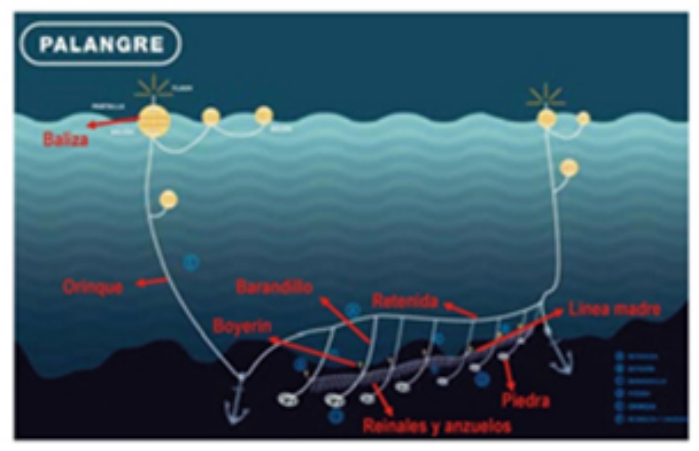

Palange tradicional

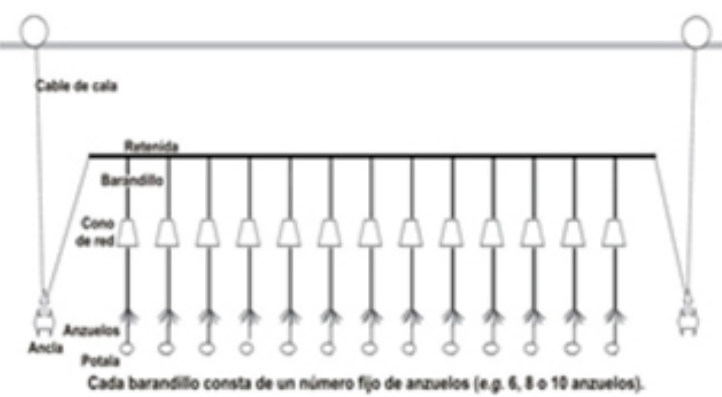

Palange cachalotero

Fig. 2. Desembarque ( $\mathrm{t}$ ) de bacalao de profundidad en aguas chilena en la flota industrial y los valores de la cuota de captura asignada al área de licitación $\left(47^{\circ}-57^{\circ} \mathrm{S}\right)$. Fuente Sernapesca, Subpesca e IFOP.

de los mamíferos sobre las capturas. Los exitosos resultados de esta modificación, en mitigar la interacción de los mamíferos, y de paso eliminar la interacción con las aves, conllevó al uso masivo de este aparejo por toda la flota palangrera fábrica hasta la actualidad.

El cambio del aparejo de pesca y la fuerte interacción de los mamíferos en las capturas del recurso ha generado una mayor incertidumbre sobre el indicador de CPUE, junto con los cambios en la dinámica de la flota para reducir los efectos de los mamíferos en sus rendimientos de pesca. Lo anterior tiene repercusiones en la indice de CPUE.

La situación mencionada en el período reciente ha significado al monitoreo un incremento en los desafíos en la toma de datos hacia el Observador Científico en bacalao de profundidad. Por ejemplo el indicador del rendimiento de pesca medido en gramos por barandillo en vez de gramos por anzuelo calado (Fig. 4), estructura de talla de las capturas según aspectos espacio-temporales (Fig. 5) y registro de la interacción de los mamíferos en la actividad de pesca (Fig. 6 y 7). Esto se ha 


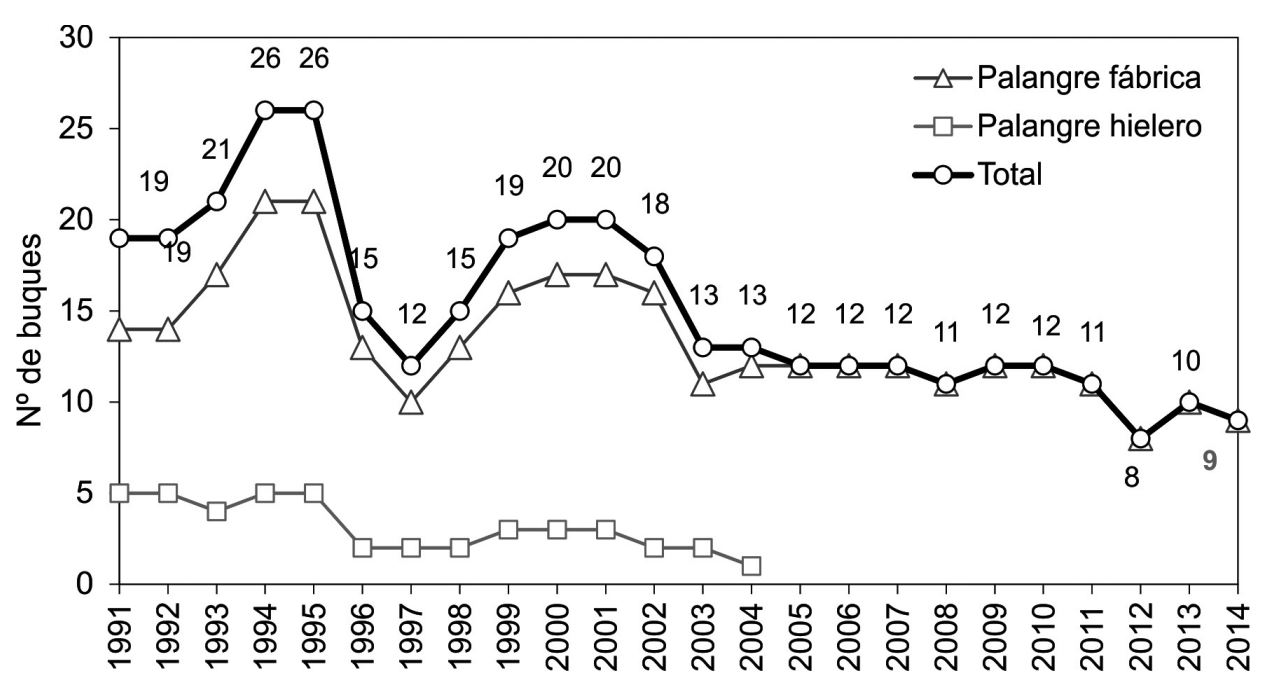

Fig. 3. Número de naves anuales en la flota palangre bacaladera por tipo de buque. Fuente IFOP.

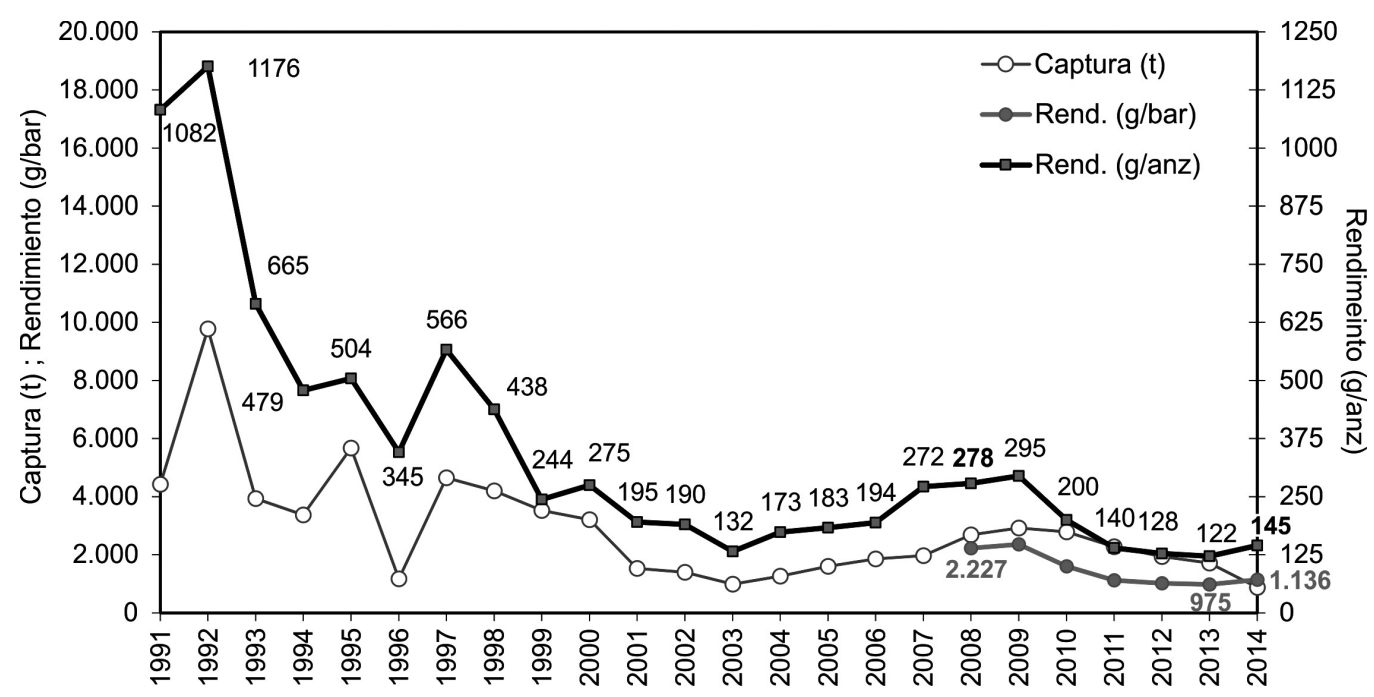

Fig. 4. Distribución del esfuerzo, captura, rendimiento de pesca por anzuelo (g/anz) por barandillo (g/bar) de bacalao de profundidad en la flota palangrera bacaladera, área de licitación entre 1991 y 2014 . Fuente IFOP.

traducido en modificar las bitácoras de pesca que incluya los registros de pesca del uso de los aparejos modificados, como también la interacción de mamíferos en las capturas y pesca incidental (aves), y a su vez siempre mejorando la cobertura de monitoreo en la flota.

El desafío hacia la toma de los datos por parte del Observador Científico ha conllevado en los últimos años fortalecer la colaboración en la toma de los datos a bordo con usuarios de la flota palangrera fábrica, orientados a registrar la interacción de los mamíferos como se muestra parte de la bitácora de pesca empleada por el Observador Científico en la Fig. 8. Por su parte, producto de la necesidad de conocer e iniciar el registro de descarte en la pesquería de bacalao de profundidad es que también se ha agregado en la toma de datos el registro de estas prácticas (Fig. 8).

Discusión de los desafíos del monitoreo de la pesquería

Como se ha descrito, la pesquería de 

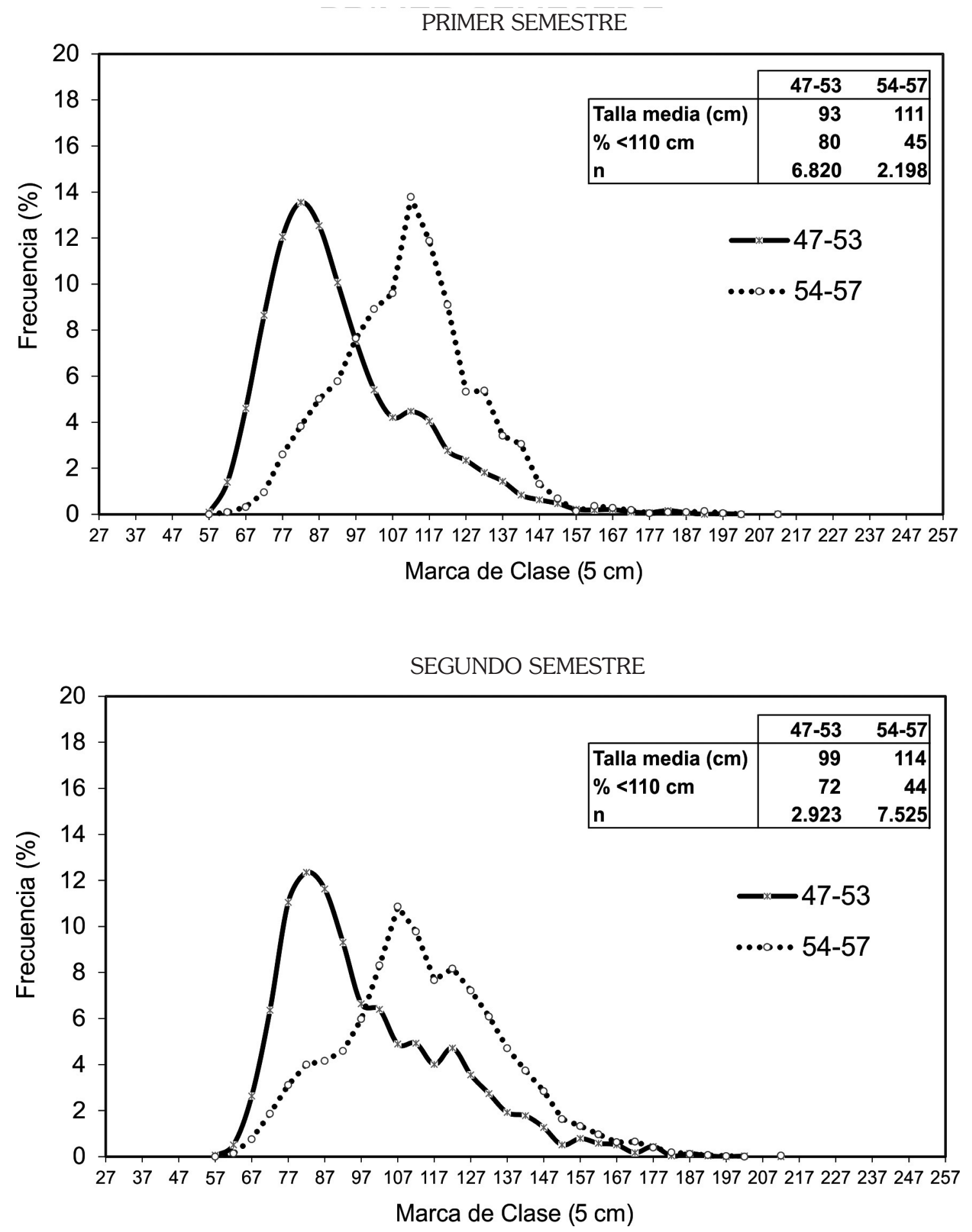

Fig. 5. Distribución de talla de bacalao de profundidad en la flota industrial palangrera de la PDA por semestre y rango de latitud $\left(47^{\circ}-53^{\circ} \mathrm{S}\right.$ y $\left.54^{\circ}-57^{\circ} \mathrm{S}\right)$ del 2014 . Fuente IFOP. 


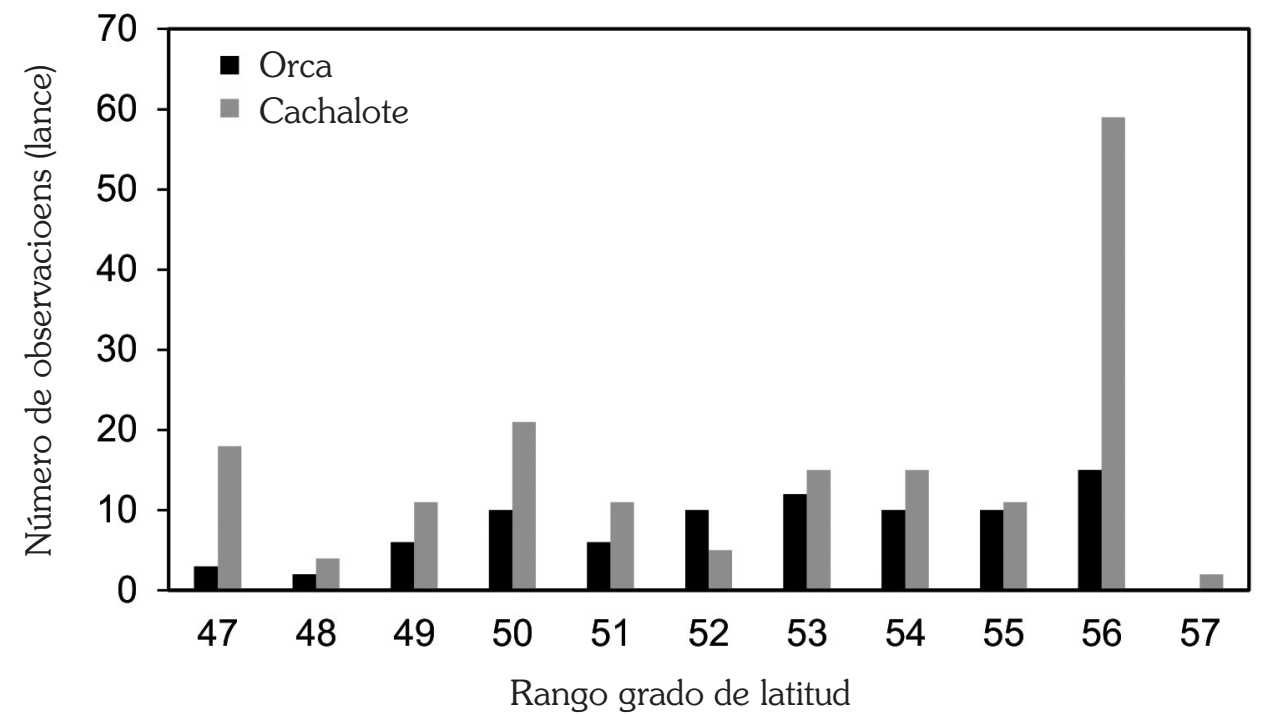

Fig. 6. Número de observaciones (o lances) con presencia de orcas y cachalotes por rango de grado de latitud en la pesquería de bacalao de profundidad en el área licitada, 2014. Fuente IFOP.

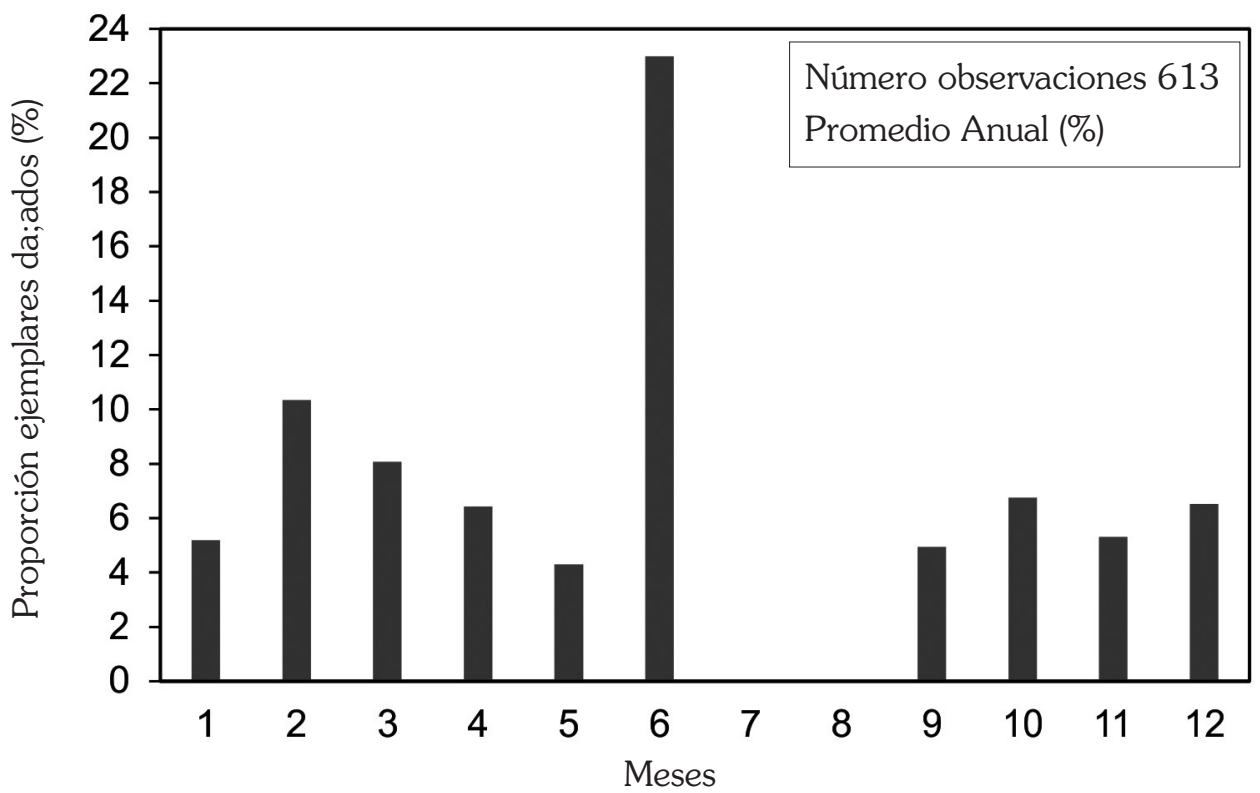

Fig. 7. Proporción (\%) mensual de ejemplares dañados por efecto de las orcas y cachalotes en la pesquería de bacalao de profundidad en el área licitada, 2014. Fuente IFOP.

bacalao de profundidad al sur del paralelo $47^{\circ}$ $\mathrm{S}$. ha contado con toma de datos pesqueros y biológicos desde los inicios de esta pesquería en los años noventa, pero la toma de datos en el programa de monitoreo se ha ido adaptando a los mayores requerimientos de conocimiento del estado situación de la pesquería, los requerimientos de evaluación de stock y en la toma de decisión de manejo de la pesquería, en donde la colaboración con los usuarios de la pesquería ha tomado mayor relevancia.

En los últimos años, los desafíos en la toma de datos por parte del Observador Científico en el programa de monitoreo de la pesquería ha 


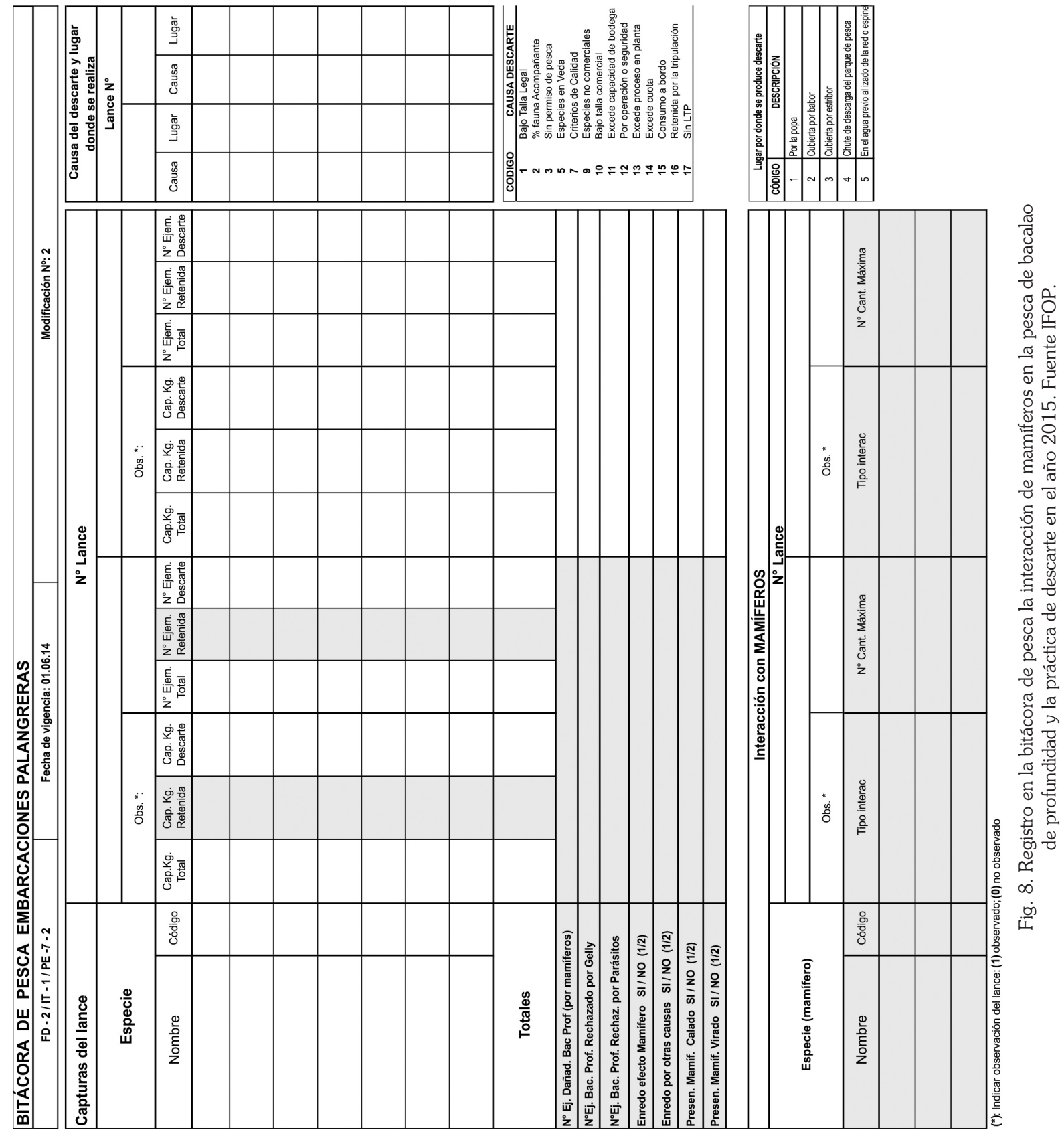


incrementado principalmente por representar la realidad de los efectos de la interacción de los mamíferos en las capturas de bacalao de profundidad, sumándose los requerimientos de registro de descarte en esta pesquería. Esto reflejado en la modificación y adecuación de la bitácora de pesca. Esta situación ha generado en algunos casos la necesidad de embarcar dos Observadores Científicos en un viaje de pesca en la flota palangrera fábrica. No obstante, este esfuerzo no es suficiente, por ello que un aspecto relevante ha sido el vínculo de colaboración con los usuarios en mejorar la toma de los datos pesqueros

Estos desafíos han abierto la oportunidad de revisar de forma más frecuente los diversos campos pesqueros que se registran en las bitácoras de pesca en el programa de monitoreo del recurso, y en esta revisión los usuarios tienen una importante participación, ya que el propósito es representar la realidad que es observada en la actividad de pesca y con ello avanzar en reducir las incertidumbres, sobre todo respecto de la interacción con los mamíferos.

No obstante, el programa de monitoreo tiene aún desafíos en el conocimiento de la pesquería de bacalao de profundidad, como por ejemplo en al menos en los siguientes temas:

- Censo de la actividad pesquera de la flota palangrera fábrica. Es decir $100 \%$ de cobertura en la flota en todo el año de operación de la pesca.

- Estudios de las capturas y esfuerzo de pesca.

- Estudios de la actividad reproductiva

- Estudios trofodinámicos.

- Estudios de marca-recaptura

- Estudios genéticos y en otras técnicas de identificación de unidades.

- Estudios de interacción con los mamíferos.

- Estudios de comportamiento de los mamíferos

El listado anterior solo intenta abrir un debate respecto de los desafíos en la toma de datos en el programa de monitoreo con objeto de mejorar la investigación sobre el recurso y su pesquería, orientadoal manejo de la pesquería y al conocimiento integral de las diferentes interrelaciones en que ella se desenvuelve. No obstante, estos desafíos $\mathrm{u}$ otros requieren de financiamiento adecuado para avanzar alcanzarlos, en donde a su vez el programa de monitoreo incorpore vínculos con otros grupos de investigadores del mundo científico.

\section{AGRADECIMIENTOS}

Los autores agradecen a los capitanes, patrones, oficiales y tripulación de las naves de pesca de las compañías Global Pesca Spa, Deris S.A. y Pesca Cisne S.A. Además se reconoce el trabajo de observadores científicos de IFOP con base en Punta Arenas.

\section{LITERATURA CITRADA}

Galleguillos, R., Ferrada, S., Hernández, C., Canales-Aguirre, C., Aedo, G., San Martín, M., \& Astete, S. (2008). Unidades poblacionales del bacalao de profundidad. FIP 2006-41. Informe Final, Universidad de Concepción.

Gálvez, P., Céspedes, R., Chong, L., San Juan, R., Ojeda, V., Meléndez, R., Molina, B.,...González, J. (2014). Proyecto 1.9: Programa de Seguimiento de las Pesquerías Demersales y Aguas Profundas, 2013. Sección VI: Recursos de Aguas Profundas, 2013. Informe Final SUBPESCA, Valparaíso, Chile, IFOP.

Young, Z., Zuleta, A., Robotham, H., Aguayo, M., \& Cid, L. (1992). Evaluación del stock de bacalao de profundidad entre las latitudes $47^{\circ} \mathrm{S}$ y $57^{\circ} \mathrm{S}$. Informe Técnico. IFOP - SUBPESCA.

Young, Z., González, H., \& Gálvez, P. (1998). Análisis de la pesquería de bacalao de profundidad en la zona sur-austral. Informe Final FIP-IFOP 96-40.

Young, Z., Oliva, J., Olivares, A., \& Díaz, E. (1999). Aspectos reproductivos del recurso bacalao de profundidad en la I a $X$ Regiones. Informe Final FIP-IFOP 9716.

Wiff, R., Young, Z., \& Quiroz, J. C. (2006). Investigación evaluación de stock CTP bacalao de profundidad sur 47ㅇ, 2006. SUBPESCA. Informe Final, IFOP. 